Die in diesem Artikel enthaltenen Bilder und sonstiges Drittmaterial unterliegen ebenfalls der genannten Creative Commons Lizenz, sofern sich aus der Abbildungslegende nichts anderes ergibt. Sofern das betreffende Material nicht unter der genannten Creative Commons Lizenz steht und die betreffende Handlung nicht nach gesetzlichen Vorschriften erlaubt ist, ist für die oben aufgeführten Weiterverwendungen des Materials die Einwilligung des jeweiligen Rechteinhabers einzuholen.

Weitere Details zur Lizenz entnehmen Sie bitte der Lizenzinformation auf http://creativecommons.org/ licenses/by/4.0/deed.de.

\title{
Schmied, Doris/Wüstenrot Stiftung (Hrsg.): Große Dörfer - Kleine Städte (RURAL, Bd. 9), 148 S., Cuvillier, Göttingen 2018.
}

\section{Lina Schröder}

Online publiziert: 22. September 2020

(C) Der/die Autor(en) 2020

Das Fazit des vorliegenden Tagungsbands vom 20. Dorfsymposium (2016) spiegelt die erfolgreiche Arbeit des 1977 gegründeten interdisziplinären Arbeitskreises für Dorfentwicklung: Als Gründungsmotiv gibt Gerhard Henkel in seinem Rückblick eine Phase des tiefgreifenden Wandels des Dorfes an (S. 135). Zur effektiven Erarbeitung aktueller Probleme wurde sich damals bewusst auf eine interdisziplinäre und an der Gegenwart orientierte Herangehensweise verständigt (S. 140). Walter Christallers Zentralorttheorie wurde dabei kritisch hinterfragt, da diese zu einem planvollen Abbau von Autonomie, Infrastruktur und Arbeitsplätzen im Zuge der Herabqualifizierung deutscher Dörfer geführt habe (S. 146).

Die weiteren sieben verständlich geschriebenen Aufsätze stammen aus der Feder von elf Autoren, unter anderem aus der Politikwissenschaft, Geografie, Soziologie und Architektur. Jeder Beitrag beginnt mit einer kurzen Zusammenfassung sowohl in deutscher als auch in englischer Sprache und wird durch Abbildungen und Tabellen in den jeweiligen Aussagen unterstützt. An der jüngsten Vergangenheit orientiert, diskutieren sie unter anderem die Folgen der Schrumpfungsprozesse in der ländlichen Bevölkerung und betonen, dass große Dörfer und kleine Städte fächerübergreifend von der Forschung vernachlässigt wurden. Eine klare Definition bezüglich der

L. Schröder $(\bowtie)$

Julius-Maximilians-Universität Würzburg, Würzburg, Deutschland

E-Mail: lina.schroeder@uni-wuerzburg.de 
Unterscheidung beider Siedlungsformen sei, so Doris Schmied und Stefan Krämer in ihrer Einleitung, kaum möglich (S. 1). Größentypologien als Instrument werden im Weiteren folgerichtig kritisch hinterfragt, da zum Beispiel ein Einwohner mehr oder weniger über die Zugehörigkeit zu den Kategorien ,Dorf“ und ,Stadt" entscheiden kann (S. 2).

Annett Steinführer weist in ihrem anschließenden Aufsatz bezüglich der Alterung und des siedlungsstrukturellen Wandels von Kleinstädten darauf hin, dass in den letzten Jahrzehnten außerdem viele Kleinstädte mit einem in früheren Epochen verliehenen Stadtrecht durch Eingemeindungen verschwunden sind, im Gegenzug jedoch neue Kleinstädte entstanden sind (S. 10).

Steffen Maretzke und Lars Porsche greifen diesen Aspekt im Folgebeitrag mit der These auf, dass sich die Vielfalt des ländlichen Raumes mit seinen unterschiedlichen Rahmenbedingungen auch in den Strukturen der zugehörigen Kleinstädte niederschlagen muss (S. 35). Als problematisch sehen sie vor allem die demografischen Schrumpfungsprozesse an, deren Folgen sie anschaulich anhand des Betriebs einer Grundschule oder des Unterhaltes eines leistungsfähigen Angebots des öffentlichen Nahverkehrs verdeutlichen: Je weniger Personen diese Infrastrukturen nachfragen, desto schwieriger gestaltet sich ein rentabler Betrieb (S. 44).

Henning Bombeck und Udo Knapp konkretisieren diese Problematik am Beispiel Mecklenburg-Vorpommerns. Als eine der Ursachen für den Veränderungsprozess sehen sie die Konsequenzen individueller Entscheidungen und Freiheiten zur Selbstverwirklichung, die mit Ansprüchen an ein hohes Bildungsniveau, einen gesicherten Wohlstand und eine immer bessere Gesundheitsversorgung einhergehen (S. 63). Als eine mögliche Lösung fordern sie, dass Entscheidungen und Strukturplanungen vor allem auch von den betroffenen Kommunen und Dörfern vor Ort zu erfolgen haben (S. 76).

Auch Karl Martin Born diskutiert den demografischen Schrumpfungsprozess. Er sieht allerdings das Hauptproblem darin, dass Dörfer und Kleinstädte von der Öffentlichkeit und der Kommunalpolitik nicht in ihrer Historizität gesehen werden (S. 84). Seine These belegt er, ebenfalls am Beispiel Mecklenburg-Vorpommerns, indem er unter anderem an die Auswirkungen des Zustroms von Flüchtlingen nach dem Zweiten Weltkrieg erinnert (S. 84).

Niklas Nitzschke widmet sich im anschließenden Aufsatz der Baukultur. Kleinstädte seien sowohl von ländlichen als auch von urbanen Siedlungsstrukturen geprägt. Das oftmals erhaltene historische Zentrum weise in der Regel eine höhere Bebauungsdichte mit Versorgungsangeboten auf, während die Erweiterungsgebiete eher locker bebaut werden (S. 92). Nicht selten sind es aber gerade die historischen Gebäude, die das Ortsbild kennzeichnen und für die Bewohner identitätsstiftend wirken. Dies müsse berücksichtigt werden (S. 98).

Den Aspekt der Baukultur greift ebenso Thomas Vielhalber am Beispiel von Arnsberg in Südwestfalen auf. Dabei geht er der Frage nach, welche Rolle Baukultur dort seit jeher und speziell in den letzten Jahren spielte und welche Ansätze sich auf andere, gerade auch kleinere Orte übertragen lassen (S. 106). Die Besonderheit seines Fallbeispiels besteht darin, dass die 74.000 Einwohner zählende Stadt im Zuge der kommunalen Neugliederung 1975 aus drei ehemals selbstständigen Städten und zwölf Dörfern entstand. Anhand verschiedener Beispiele beleuchtet Vielhalber 
diverse Baumaßnahmen und das seiner Meinung nach gelungene Ineinandergreifen verschiedener Instanzen. In seinem Fazit hält er bezüglich seiner Ausgangsfrage fest, dass sich das Arnsberger Baumodell durchaus auf andere Orte übertragen lässt (S. 121).

Ein ebenfalls überaus gelungenes Stadtkonzept stellt im Anschluss Thomas Herker mit Pfaffenhofen an der Ilm vor. Der Stadt wurde im Rahmen eines Wettbewerbs der Titel „lebenswerteste Kleinstadt der Welt 2011“ verliehen (S. 124). Aufgrund ihrer privilegierten Lage zwischen München und Ingolstadt hatte sie mit einer starken Zunahme der Stadtbevölkerung zu kämpfen. Dieser versucht die Stadt über die Schaffung erschwinglichen Wohnraums ohne Landschaftszersiedelung, durch Nachhaltigkeit bei der Daseinsvorsorge und Infrastruktur sowie durch Lebensqualität in allen Lebenslagen zu begegnen (S. 124). Die Verwendung von 100 Prozent regenerativer Energien und konsequent wohngiftfreier Baustoffe machen das ecoQuartier zum Vorreiter (S. 126).

Alle Autoren des Sammelbandes sind sich einig, dass viele aktuelle Prozesse nur über die historische Vergangenheit einer Ortschaft verständlich werden - eine Erkenntnis, die jedem sich mit Stadt- und Dorfgeschichte befassenden Historiker die Lektüre des Bandes empfiehlt, auch wenn die Rezensentin einen Beitrag aus der Feder eines tatsächlichen Historikers vermisst.

Funding Open Access funding provided by Projekt DEAL.

Open Access Dieser Artikel wird unter der Creative Commons Namensnennung 4.0 International Lizenz veröffentlicht, welche die Nutzung, Vervielfältigung, Bearbeitung, Verbreitung und Wiedergabe in jeglichem Medium und Format erlaubt, sofern Sie den/die ursprünglichen Autor(en) und die Quelle ordnungsgemäß nennen, einen Link zur Creative Commons Lizenz beifügen und angeben, ob Änderungen vorgenommen wurden.

Die in diesem Artikel enthaltenen Bilder und sonstiges Drittmaterial unterliegen ebenfalls der genannten Creative Commons Lizenz, sofern sich aus der Abbildungslegende nichts anderes ergibt. Sofern das betreffende Material nicht unter der genannten Creative Commons Lizenz steht und die betreffende Handlung nicht nach gesetzlichen Vorschriften erlaubt ist, ist für die oben aufgeführten Weiterverwendungen des Materials die Einwilligung des jeweiligen Rechteinhabers einzuholen.

Weitere Details zur Lizenz entnehmen Sie bitte der Lizenzinformation auf http://creativecommons.org/ licenses/by/4.0/deed.de. 